\title{
Structural optimization design on composite cantilever-beam-type actuator withTbDyFe alloy
}

\author{
YuluZeng ${ }^{1,2}$, ZhifangZhu ${ }^{1,2},{\text { Bin } \mathrm{Xu}^{1,2}, \text { QuanguoLu }}^{1,2}$, Cuiqun $\mathrm{He}^{1,2}$, Hongmin \\ Wen $^{1,2}$,Bin Zeng ${ }^{2}$ and Ya Cha ${ }^{2}$
}

1Jiangxi Province Key Laboratory of precision drive and control,Nanchang,330099,China

2Nanchang Institute of Mechanical and Electrical Engineering,Nanchang,330099,China

Keywords: TbDyFe; composite cantilever beam; optimization design.

\begin{abstract}
Acomposite cantilever-beam-type actuator and its driving structure were designed byemployingTbDyFe alloy in this paper after the introduction aboutthe working principle of actuator. Magnetic circuit analysis and the number and position of the Terfenol-D slice adhesion are mainly included for the designed actuator. Then simulations on the magnetic circuit of the designed actuator and its driving structures were carried on by using the software of COMSOL Multipysics. Finally, the structure of the actuator was optimized based on the simulation results. It indicates that the actuator with optimized structure has more excellent performance.
\end{abstract}

\section{Introduction}

Composite cantilever beam is a intelligent device byconglutinating the functional materials with the mechanical cantilever beam. It can be applied in many areas such as the vibration active control [1], the force sensor [2], and the light reflex positioning[3]. Piezoelectric material, magnetic control shape memory alloy, and magnetostriction material are the three most widely applied functional materials which are the ideal materials for the construction of the composite cantilever beam.

\section{Common functional materials}

TbDyFe is of a new magnetostriction alloy which has advantages such as large strain, high precision, brute force, fast response, and high reliability. By applying the magnetic driving technique, it can drive without wire [4]. Terfenol-D with Tb0.27Dy1.073Fe1.93 as its main composition owns many advantages over the piezoelectric material PZT. For example, the Terfenol-D dilatation coefficient is over one order of magnitude of the PZT, and its response speed is more prompt and its energy conversion efficiency is higher.

About the design of TbDyFe composite cantilever beam, Na et al. [5] obtained the rigorous solution which is suitable for the bending problem of magnetic film-substrate system under point load at free ends with any thickness ratio of the magnetic film to the substrate based on the fundamental mechanical equilibrium equations. They also finished the design and optimization of the micro actuator cantilever beam based on the materials geometric and physical parameters.

\section{Working principle of cantilever-beam-type actuator}

The basic working principle of the cantilever-beam-type actuator is shown in Fig.1.Many Terfenol-D slices are continuously glued on the substrate to drive.Bending deformation will be produced due to the shrink of the top surface of the substrate which is closely glued with the Terfenol-D slice. The magnitude of the bending is determined by the magnetic field intensity which enables the precision control of the open amount of the actuator through controlling the magnetic field. 


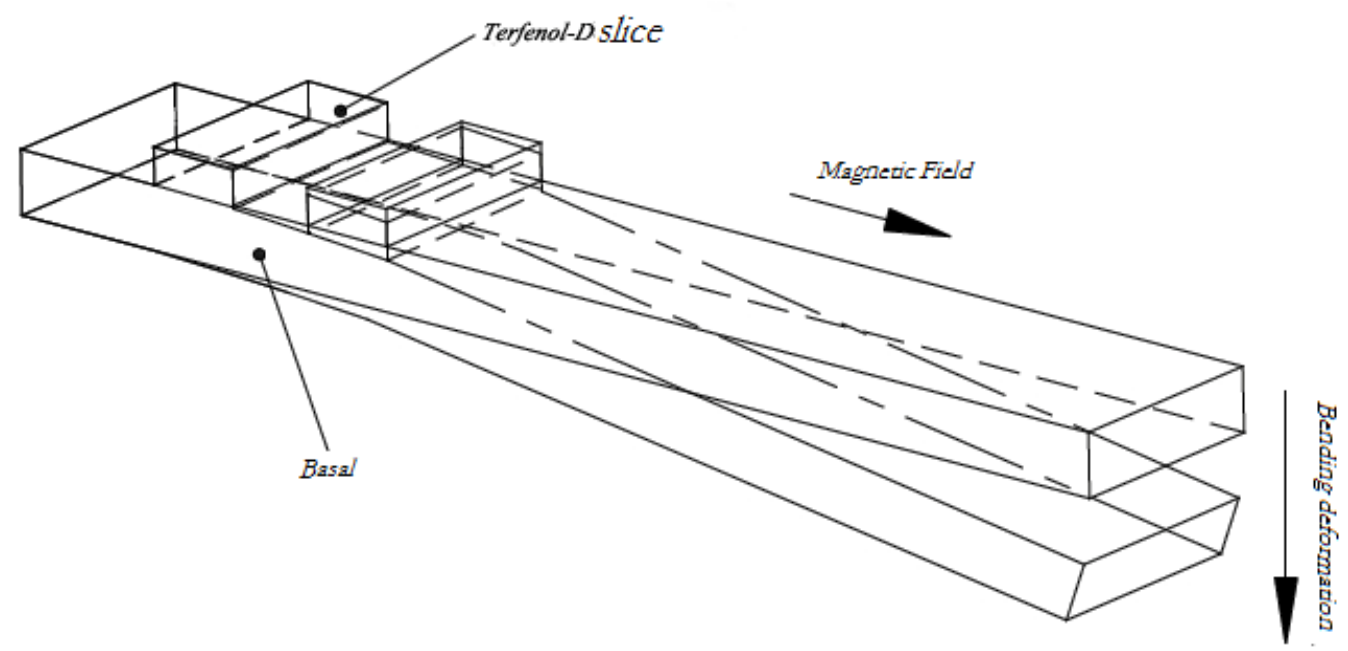

Fig. 1 Schematic of actuator working principle

\subsection{Structural design of actuator}

A beryllium bronze after heat treatment was processed by wire-electrode cutting to be the substrate with the dimension of $82 \times 9.5 \times 0.55 \mathrm{~mm}$. Thus the dimensions of both the Terfenol-D slices and the actuator were determined. The maximum elongation $\Delta \operatorname{lmax}$ of the Terfenol-D slices depend on their length IT along magnetization direction. The linear magnetostriction coefficient of the super magnetostriction materials, $\lambda$, can be described as,

$$
\lambda=\Delta l / l
$$

where, $l$ is the length of materials, and $\Delta l$ denotes the variation of the length.

According to Eq. (1), the maximum elongation of the Terfenol-D slice along magnetization directioncan be calculated as,

$$
\Delta l_{\max }=\lambda_{s} \cdot l_{T}
$$

where the symbol $\lambda_{\mathrm{s}}$ represents saturation magnetostriction coefficient.

The diagram showing the working principle of the actuator is depicted as Fig.2. It shows the deformation will reach maximum when the Terfenol-D slice is up to the maximum deformation.

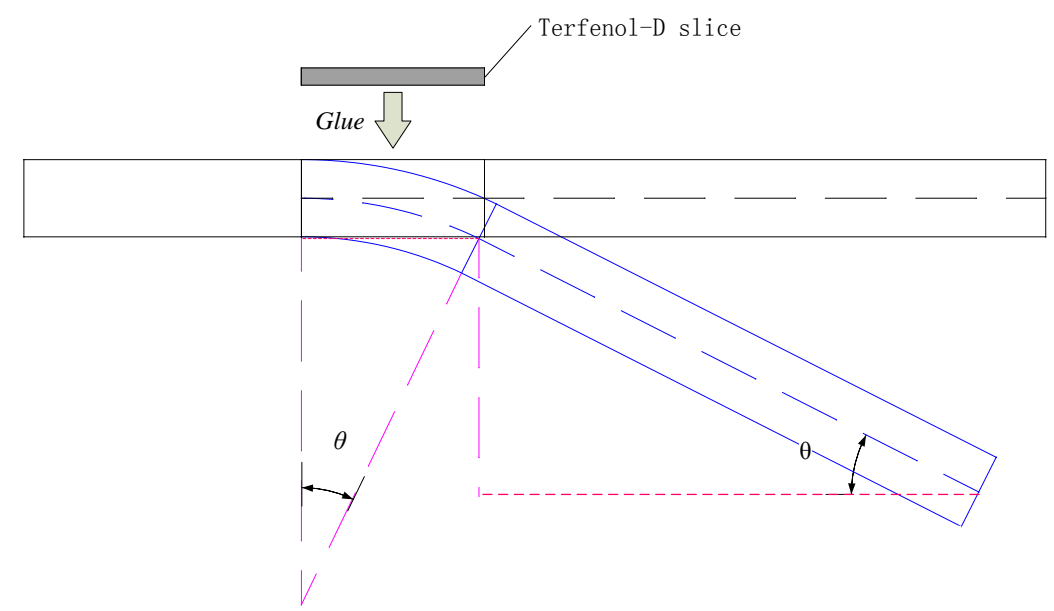

Fig. 2 Schematic of the actuator

\subsection{Magnetism-mechanical coupling model.}

The kinetic equation describing the three dimensional distribution of the driving magnetic field was derived by employing the Maxwell equation set in order to establish the three dimensional nonlinear model of the composite cantilever beam. The differential Maxwell equation set can be depicted as [6], 


$$
\begin{aligned}
& \nabla \times \mathrm{E}=-\frac{\partial B}{\partial t} \\
& \nabla \times \mathrm{H}=\mathrm{J}+\frac{\partial \mathrm{D}}{\partial t} \\
& \nabla \bullet B=0 \\
& \nabla \bullet E=\frac{P}{\varepsilon_{0}}
\end{aligned}
$$

where, the symbol $P$ denotes the spatial electric density, $\varepsilon_{0}$ represents the permittivity of vacuum. The electric displacement vector, $D$, can be neglected when the driving frequency is lower than 30 $\mathrm{MHz}$. The total current in Eq. consists of two parts, namely the source current density $\left(J_{\mathrm{s}}\right)$ and the external current density $\left(J_{\mathrm{e}}\right)$ produced by eddy current effect. Thus the divergence of the magnetic field intensity can be shown as,

$$
\nabla \times H=J_{s}+J_{e}
$$

Assuming the variables in the magnetic field system to be solved as the vector magnetomotive force $A\left(A_{x}, A_{y}, A_{z}\right)$, the electric field intensity can be calculated by the vector magnetomotive force, which is derived as,

$$
E=-\frac{\partial A}{\partial t}-\nabla \phi
$$

where $\mathrm{A}$ is the vector magnetomotive force, $\emptyset$ is the scalar potential function, the eddy current can be calculated as,

$$
J_{e}=\sigma_{e} E=\sigma_{e}\left(-\frac{\partial A}{\partial t}-\nabla \phi\right)=\sigma_{e}\left(-\frac{\partial A}{\partial t}-0\right)=-\sigma \frac{\partial A}{\partial t}
$$

where $\sigma_{\epsilon}$ is the material conductivity. The magnetic induction intensity is described by the vector magnetic potential as,

$$
B=\nabla \times A
$$

\subsection{Magnetic circuit design of driving structure.}

The maximum magnetic field strength is at the inside of the inner diameter area of the cylinder coil. Consequently, putting the Terfenol-D slices into this area could improve the using efficiency of energy. The driving system mainly consists of coil, coil former, core assembly (being placed in the skeleton to fix a segment of the valve block), shell, and end cap.

Some principles should be followed during the design of magnetic circuit in order for better magnetism-mechanical transfer efficiency of the actuator. They are,

(1) Proper components should be selected to form the magnetic circuit of the actuator, which enables bigger flux in the magnetic return path and more magnetic lines of force through the Terfenol-D driving slices.

(2)Magnetic leakage should be reduced to make maximize the flux through the driving slices. The schematic of the driving structure and its magnetic return path is shown in Fig. 3.

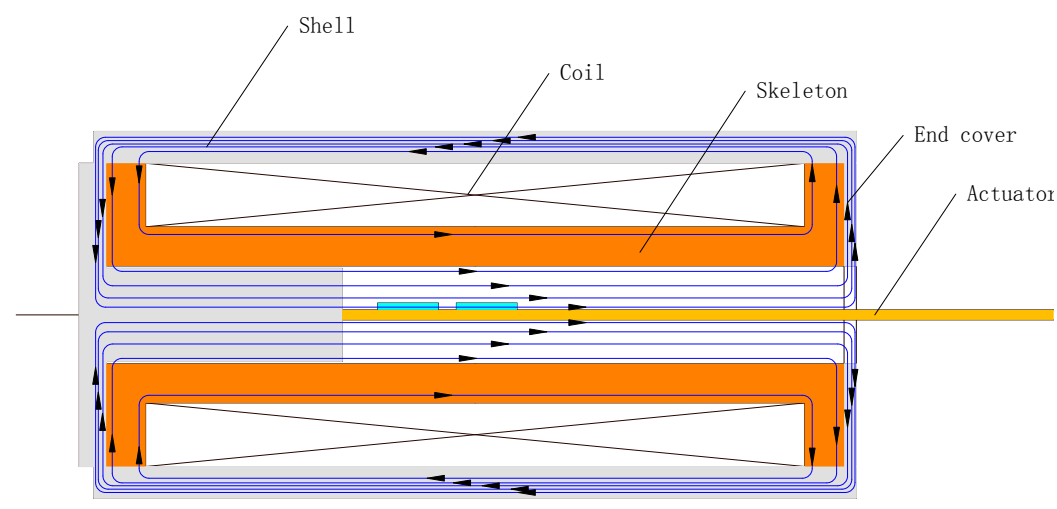

Fig. 3 Schematic of driving structure and its magnetic return path 


\subsection{Finite element analysis on magnetic field.}

The popular software COMSOL Multiphysics is employed in this paper to simulate the magnetic field of the designed actuator model. The two-dimensional axial symmetry module [7] is applied in the finite element simulation under the consideration that the driving coil structure is of three-dimensional axial symmetry in order to reduce the computational effort and save the computer memory to improve the calculation accuracy. The established two-dimensional axial symmetry model is illustrated in Fig. 4(a), and its finite element model can be obtained as Fig. 4(b). When an electric current density of $2 \mathrm{~A} / \mathrm{mm} 2$ is applied to the coil area, the simulation results are obtained which are shown in Figs. 4 (c) and (d).
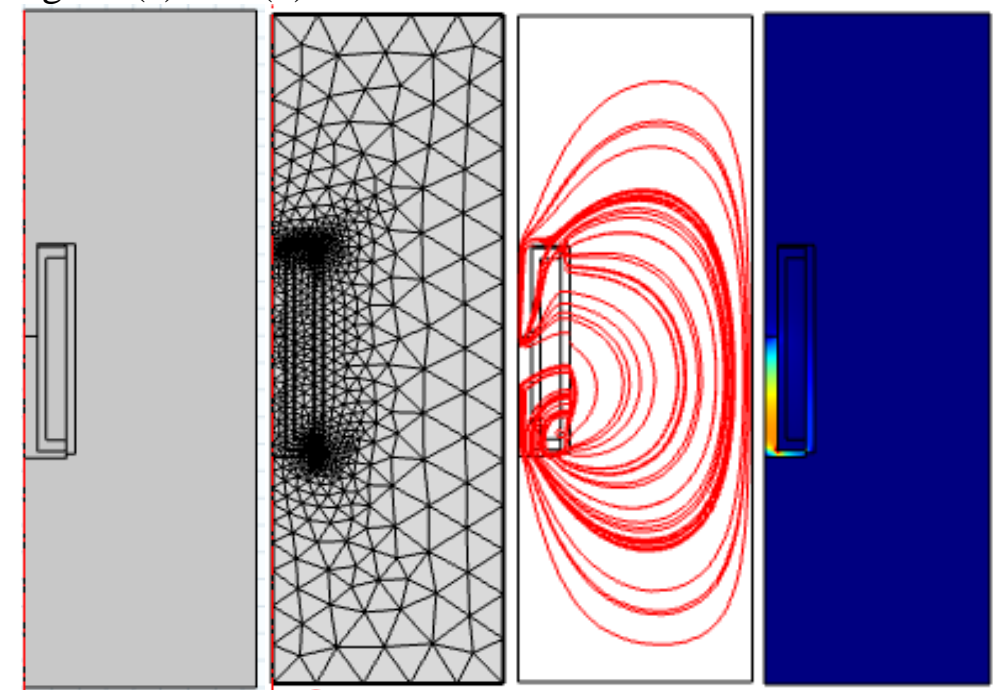
(a)Geometric model (b)Mesh generation (c) Distribution of magnetic line of force (d) Contour of magnetic flux

Fig. 4 Magnetic simulation of driving structure

\section{Optimum structural design}

Through the aforementioned analysis it indicates that many unreasonable designs also exist at present, for example, excessive driving coil length and relative smaller space for the Terfenol-D slices will not only enlarge the space occupied by the device but also increase the whole length of the actuator. In order to overcome these shortages, an improved actuator and its driving structure is developed based on the theoretical analysis in this paper. Its structural diagram is shown in Fig. 5. In the improved actuator, the offset coils are added, and the driving cylinder height is up to $115 \mathrm{~mm}$ and its external diameter is $95 \mathrm{~mm}$; the total number of turns of the offset coils is 1180 while that of the driving coils is 680. Considering that magnetic field near the coil center is strong and uniform, the beryllium bronze is selected to be processed as the valve block with the dimension (thickness $\times$ length $\times$ width) of $1.5 \times 85 \times 8.5 \mathrm{~mm}$. It is noted that clearance of $2.5 \mathrm{~mm}$ should be set between the five Terfenol-D slices with dimension of $6 \times 3 \times 1 \mathrm{~mm}$ and the fixed ends for the convenience of gluing. 


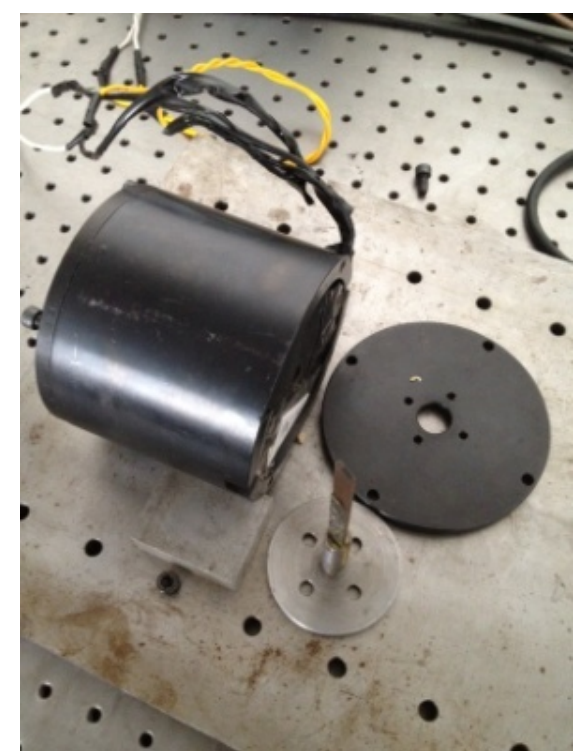

Fig. 5 Improved actuator and its driving structure

\section{Conclusion}

An improved actuator structure was obtained through the optimization design based on the analysis results from the work done in this paper where the working principle of the composite cantilever beam actuator was studied, the magnetic circuit was analyzed, the number and positions of the Terfenol-D slices are calculated, and the electromagnetism loopsof the actuator and the driving structure are simulated by employing the finite element software.The results indicate that the structure and the performance of the improved actuator are more excellent by adding the offset coils.

\section{Acknowledgement}

This paper is finacially supported by the Youth Science Fund of Nanchang Institute of Technology(Grant No. 2012KJ009 and2012KJ010), scientific research fund of JiangXi provincial education department(Grant No. JJ13765,GJJ11628), the science and technology project (No.20122BBE500033) of Jiangxi provincial department of science and technology of China, the science and technology project(2012-KJZC-GY-JDYTH-003) of Nanchang bureau of science and technology of China and the science and technology landing project of high school in JiangXi province(Grant No.KJLD14094).

\section{References}

[1]JIA Zhenyuan, LIU Wei, ZHANG Yongshun, WANG Fuji,GUODongming. Nonlinear deformation an alysis and experiment of giant magnetostricive thin film cantilever. ChineseJournal of Mechanical Engineering, 2007, 43(12): 5-11.

[2] Supratik Datta, Jayasimha Atulasimha, Alison B Flatau. Modeling of magnetostrictive Galfenol sensor and validation using four point bending test. Journal of Applied Physics. 2007, 101(9): 09C521

[3] CAI Yu-li ,DING Gui-fu,DAI Xu-han,ZHANG Dan. Development of 1x16 MEMS optical fiber connector array. Transducer and Microsystem Technologies, 2007, 26(7): 87-89

[4] Zhang Yongshun, Wang Huiying, Zhang Ruixia, Gou Rui, Jia Zhenyuan. Bidirectional moving principle of a wireless micro robot based on giant magnetostriction actuator. IEEE International Conference on Robotics and Biomimetics, IEEE ROBIO 2004: 113-118.

[5] NA Ri-su YUN Guo-hong RONG Jian-hong. Designand optimization of the magnetostrictive film substrate cantilevermicroactuators.Scientia Sinica Technologica, 2007,37(7): 
914 922.

[6] MA Xikui. Electromagnetic theory and the applications[M]. Xi'an: Xi'an Jiaotong University Press,2000.

[7] Ma Hui,Wang Gang.Basic operation guide and answers to common problems ofCOMSOL Multiphysics[M]. China Communications Press,2009. 